Bull. Austral. Math. Soc.

$42 \mathrm{~B} 25,42 \mathrm{~B} 20$

VOL. 66 (2002) [75-90]

\title{
WEIGHTED ESTIMATES FOR FRACTIONAL MAXIMAL FUNCTIONS RELATED TO SPHERICAL MEANS
}

\author{
Michael Cowling, José García-Cuerva and Hendra Gunawan
}

We prove weighted $L^{p}-L^{q}$ estimates for the maximal operators $\mathcal{M}^{\alpha}$, given by $\mathcal{M}^{\alpha} f$ $=\sup _{t>0}\left|t^{\alpha} \mu_{t} * f\right|$, where $\mu_{t}$ denotes the normalised surface measure on the sphere of centre 0 and radius $t$ in $\mathbf{R}^{d}$. The techniques used involve interpolation and the Mellin transform. To do this, we also prove weighted $L^{p}-L^{q}$ estimates for the operators of convolution with the kernels $|\cdot|^{-\alpha-i \eta}$.

\section{INTRODUCTION}

Denote by $\mu_{t}$ the normalised surface measure on the sphere in $\mathbf{R}^{d}$ with centre 0 and radius $t$. We shall be interested in weighted $L^{p}-L^{q}$ estimates for the maximal operators $\mathcal{M}^{\alpha}$, given by the formula

$$
\mathcal{M}^{\alpha} f=\sup _{t>0}\left|t^{\alpha} \mu_{t} * f\right|
$$

whenever this makes sense. Unweighted estimates for this operator were proved by Ober$\operatorname{lin}[\mathbf{1 1}]$.

One of the reasons why we are concerned with these estimates is the following. We know that $\mu_{t}$ is $L^{p}$-improving, that is, for some (and hence all) $p$ in $(1, \infty)$, there exists $q$ in $(p, \infty)$ and a constant $C_{p, q, t}$ such that

$$
\left\|\mu_{t} * f\right\|_{q} \leqslant C_{p, q, t}\|f\|_{p} \quad \forall f \in L^{p}
$$

Considerations of homogeneity show that $C_{p, q, t}=C_{p, q} t^{-\alpha}$, where $\alpha / d=1 / p-1 / q$. It follows that, for all $f$ in $L^{p}$,

$$
\left\|t^{\alpha} \mu_{t} * f\right\|_{q} \leqslant C_{p, q}\|f\|_{p} \quad \forall t \in \mathbf{R}^{+}
$$

in particular this implies that $t^{\beta} \mu_{t} * f \rightarrow 0$ in $L^{q}$ as $t \rightarrow \infty$ whenever $0 \leqslant \beta<\alpha$.

Received 7th January, 2002

The work was initiated when the second and the third authors visited the Department of Pure Mathematics, University of New South Wales, Sydney, Australia, in March 1998. The first and third authors then visited the Departamento de Matemáticas, Universidad Autónoma de Madrid, Spain, in September 1998 and May 2000, to finish the paper. The hospitality of both institutions is greatly appreciated.

Copyright Clearance Centre, Inc. Serial-fee code: 0004-9727/02 \$A2.00+0.00. 
All our function spaces will be on $\mathbf{R}^{\boldsymbol{d}}$, and we omit reference to $\mathbf{R}^{\boldsymbol{d}}$ in the notation. Take $f$ in $L^{p}$, where $d /(d-1)<p \leqslant \infty$. The facts that $\mu_{t} * f \rightarrow 0$ in $L^{q}$ as $t \rightarrow \infty$ and that Stein's spherical maximal function $\mathcal{M} f=\sup _{t>0}\left|\mu_{t} * f\right|$ is in $L^{p}$ allow us to conclude that $\mu_{t} * f \rightarrow 0$ pointwise as $t \rightarrow \infty$. It is now reasonable to ask how fast this convergence is. One corollary of our work is that $\mathcal{M}^{\alpha} f$ is in $L^{q}$, so that the pointwise convergence is faster than $t^{-\beta}$ for any $\beta$ in $(0, \alpha)$.

We recall in Section 2 a few well-known results about the Muckenhoupt weight space $A_{p}$, and then in Section 3, we first review and then improve the known $L^{p}-L^{p}$ estimates involving $A_{p}$ weights for the spherical maximal function. In Section 4, we prove some weighted estimates for the convolution kernels $|\cdot|^{-\alpha-i \eta}$, where $0<\alpha<d$ and $\eta$ is real. Finally, we discuss the weighted $L^{p}-L^{q}$ estimates for the fractional maximal functions related to spherical means in Section 5 .

Constants will usually be denoted by $C$ and $\varepsilon$; the former constants may be very large, and the latter very small. These "constants" may vary from one occurrence to the next. However, they are always positive.

\section{2. $A_{p}$ WEIGHTS}

A nonnegative locally integrable function $w$ on $\mathbf{R}^{d}$ is said to belong to the Muckenhoupt class $A_{1}$ when there exists a constant $C$ such that

$$
\frac{1}{|Q|} \int_{Q} w(x) \mathrm{d} x \leqslant C w(y) \quad \text { for almost every } y \in Q
$$

for all cubes $Q$ in $\mathbf{R}^{d}$, where $|Q|$ denotes the Lebesgue measure of $Q$. If $1<p, q<\infty$, then $w$ is said to belong to $A_{p}$ when there exists a constant $C$ such that

$$
\left(\frac{1}{|Q|} \int_{Q} w(x) \mathrm{d} x\right)\left(\frac{1}{|Q|} \int_{Q} w(x)^{-1 /(p-1)} \mathrm{d} x\right)^{p-1} \leqslant C
$$

for all cubes in $\mathbf{R}^{d}$; more generally, $w$ is said to belong to $A(p, q)$ when there exists a constant $C$ such that

$$
\left(\frac{1}{|Q|} \int_{Q} w(x)^{q} \mathrm{~d} x\right)^{1 / q}\left(\frac{1}{|Q|} \int_{Q} w(x)^{-p^{\prime}} \mathrm{d} x\right)^{1 / p^{\prime}} \leqslant C
$$

for all cubes in $\mathbf{R}^{d}$. It is easy to see that $w \in A_{q / p^{\prime}+1}$ if and only if $w^{1 / q} \in A(p, q)$.

Much is known about $A_{p}$ weights. A key property is that $w \in A_{p}$ if and only if there exist $w_{1}$ and $w_{2}$ in $A_{1}$ such that $w=w_{1} w_{2}^{1-p}$. Another is that if $w \in A_{1}$, then there exists $\varepsilon_{0}$ in $\mathbf{R}^{+}$such that $w^{\theta} \in A_{1}$ for all $\theta$ in $\left[0,1+\varepsilon_{0}\right)$. This implies a similar property for $A_{p}$ weights, and also implies that, if $w \in A_{p}$ where $p>1$, then $w \in A_{q}$ for all $q$ in an interval of the form $(r, \infty)$, where $1 \leqslant r<p$. It is known that $|\cdot|^{a} \in A_{1}$ if and only if $-d<a \leqslant 0$. 
We write $L^{p}(w)$ for the space of all measurable functions $f: \mathbf{R}^{\mathbf{d}} \rightarrow \mathbf{C}$ for which $\|f\|_{p, w}$ is finite, where

$$
\|f\|_{p, w}=\left(\int_{\mathbf{R}^{d}}|f(x)|^{p} w(x) \mathrm{d} x\right)^{1 / p},
$$

with functions identified when they agree almost everywhere.

We recall the following interpolation theorem of Stein and Weiss.

Theorem 2.1. Suppose that $v$ and $w$ are weights, and that $1 \leqslant p, q \leqslant \infty$. The complex interpolation space $\left[L^{p}(v), L^{q}(w)\right]_{\theta}$ is the weighted space $L^{r}\left(v^{(1-\theta) r / p} w^{\theta_{r} / q}\right)$, where $1 / r=(1-\theta) / p+\theta / q$.

Proof: See, for example, [17, p. 211].

Let $m_{t}$ denote the normalised Lebesgue measure on the (solid) ball in $\mathbf{R}^{d}$ with centre 0 and radius $t$. For $\alpha$ in $[0, d]$, the fractional maximal operator $\mathcal{M}_{1}^{\alpha}$ is given by

$$
\mathcal{M}_{1}^{\alpha} f=\sup _{t>0}\left|t^{\alpha} m_{t} * f\right| .
$$

This operator was considered by Sawyer $[\mathbf{1 3}, \mathbf{1 4}]$. Observe that $\mathcal{M}_{1}^{0}$ is the well-known maximal operator of Hardy and Littlewood [7]. It was shown by Muckenhoupt [9] that, when $1<r<\infty$,

$$
\left\|\mathcal{M}_{1}^{0} f\right\|_{r, w} \leqslant C\|f\|_{r, w} \quad \forall f \in L^{r}(w),
$$

if and only if $w \in A_{r}$. On the other hand, we also have the trivial estimate

$$
\left\|\mathcal{M}_{1}^{d} f\right\|_{\infty} \leqslant C\|f\|_{1} \quad \forall f \in L^{1},
$$

where $C^{-1}$ is the measure of the unit ball in $\mathbf{R}^{d}$. It follows from Theorem 2.1 that, if $1 \leqslant p<q \leqslant \infty$ and $\alpha=d / p-d / q$, then

$$
\left\|\mathcal{M}_{1}^{\alpha} f\right\|_{q, w} \leqslant C\|f\|_{p, w^{p / q}} \quad \forall f \in L^{p}\left(w^{p / q}\right),
$$

for all $w$ in $A_{q / p^{\prime}+1}$. Alternatively, we may write

$$
\left\|\mathcal{M}_{1}^{\alpha} f\right\|_{q, w^{q}} \leqslant C\|f\|_{p, w^{p}} \quad \forall f \in L^{p}\left(w^{p}\right),
$$

whenever $w \in A(p, q)$. For these results, and more about weights, see the works cited above and; for example, $[6,8,10]$.

We shall prove families of weighted inequalities of the form

$$
\|\mathcal{T} f\|_{q, u} \leqslant C\|f\|_{p, u^{p / q}} \quad \forall f \in L^{p}\left(u^{p / q}\right)
$$

and

$$
\|T f\|_{q, v} \leqslant C\|f\|_{p, v p / q} \quad \forall f \in L^{p}\left(v^{p / q}\right)
$$


for all $u$ in $A_{r}^{\gamma}$ and $v$ in $A_{s}^{\delta}$. The first of these inequalities holds for weights of the form $\left(u_{1} u_{2}^{1-r}\right)^{\gamma}$ and the second holds for weights of the form $\left(v_{1} v_{2}^{1-s}\right)^{\delta}$, where $u_{1}, u_{2}, v_{1}$ and $v_{2}$ are in $A_{1}$. If, say, $\gamma \leqslant \delta$ and $\gamma(r-1) \leqslant \delta(s-1)$, then the second inequality is stronger than the first. On the other hand, if, say, $\gamma>\delta$ and $\gamma(r-1)<\delta(s-1)$, then the inequalities are incomparable. It would be interesting to know whether there is a simple way to unify two such incomparable inequalities. Clearly, if the two inequalities above hold, then interpolation gives a range of intermediate results. The inequalities also hold for a weight which is the maximum of weights in $A_{r}^{\gamma}$ and $A_{s}^{\delta}$.

\section{The SPherical MaXimal function}

In this section, we first review and then improve the known results involving $A_{p}$ weights for the spherical maximal operator on weighted $L^{p}$ spaces.

3.1. Review. The study of the spherical maximal operator $\mathcal{M}$ began when Stein [16] proved the inequality

$$
\|\mathcal{M} f\|_{p} \leqslant C\|f\|_{p} \quad \forall f \in L^{p}
$$

for the case where $d \geqslant 3$ and $p>d /(d-1)$. Much later Bourgain [1] treated the case where $d=2$ and $p>2$. This case is much more difficult, and we shall henceforth assume that $d \geqslant 3$.

Rubio de Francia [12] showed that the weighted estimate

$$
\|\mathcal{M} f\|_{p,||^{a}} \leqslant C\|f\|_{p,\left.|\cdot|\right|^{a}} \quad \forall f \in L^{p}\left(|\cdot|^{a}\right)
$$

holds when $d /(d-1)<p \leqslant \infty$ and $0 \leqslant a<d p-d-p$ (see also [6, p. 571]). Recently, Duoandikoetxea and Vega [3] and Gunawan [4] showed that similar estimates hold for more general weights. In particular, when $d /(d-1)<p \leqslant 2$, the inequality

$$
\|\mathcal{M} f\|_{p, w} \leqslant C\|f\|_{p, w} \quad \forall f \in L^{p}(w)
$$

holds for all $w$ in $A_{p}^{\gamma}$ when $\gamma<\left(d-p^{\prime}\right) / d$ (see $[3,4]$ ) and for all $w$ in $A_{1}^{\delta}$ when $\delta<\left(d-p^{\prime}\right) /(d-1)$ (see [3]). Since if $w \in A_{p}$, then $w^{1+\varepsilon} \in A_{p}$ for some positive $\varepsilon$, the same results actually hold when $\gamma=\left(d-p^{\prime}\right) / d$ and $\delta=\left(d-p^{\prime}\right) /(d-1)$. These imply that (3.1) holds when $w=|\cdot|^{a}$ and

$$
d\left(p^{\prime}-d\right) /(d-1)<a<d p-d-p .
$$

For power weights $|\cdot|^{a}$, Duoandikoetxea and Vega [3] showed that (3.1) holds when

$$
1-d<a<d p-d-p
$$

this result is sharp except perhaps when $a=1-d$. It is apparent that the results for general $A_{p}$ weights are not strong enough to imply this result. 
3.2. NEW $L^{p}-L^{p}$ ESTIMATES. As observed in [5], estimate (3.1) may be proved using interpolation. We shall generalise the method of [5] to extend the class of weights for which estimate (3.1) is valid. Define the operator $\mathcal{M}_{z}$ by

$$
\mathcal{M}_{z} f=\sup _{t>0}\left|m_{z, t} * f\right|,
$$

where (as in [16]) $m_{z, t}(x)=t^{-d} m_{z}(x / t)$ and

$$
m_{z}(x)=\Gamma(z)^{-1}\left(\left(1-|x|^{2}\right)_{+}\right)^{z-1}
$$

( $t_{+}$is equal to $t$ if $t$ is positive and 0 otherwise). This must be interpreted by analytic continuation when $\operatorname{Re} z \leqslant 0$. When $z=0$, we obtain a multiple of the spherical maximal operator: $\omega_{d-1} \mathcal{M}=2 \mathcal{M}_{0}$. Thus results for $\mathcal{M}_{0}$ imply results for $\mathcal{M}$, and vice versa.

THEOREM 3.1. Suppose that $d /(d-1)<p<\infty$ and that $\max \{0,1-p / d\}$ $\leqslant \delta \leqslant(d-2) /(d-1)$. Suppose also that $w$ is of the form $u_{1}^{\delta} u_{2}^{\delta(d-1)-(d-2)}$, where $u_{1}$ and $u_{2}$ lie in $A_{1}$. Then there exists a constant $C$ such that

$$
\left\|\mathcal{M}_{0} f\right\|_{p, w} \leqslant C\|f\|_{p, w} \quad \forall f \in L^{p}(w) .
$$

Proof: We first outline the proof. We start with three inequalities, namely

$$
\left\|\mathcal{M}_{z} f\right\|_{2} \leqslant C_{z}\|f\|_{2} \quad \forall f \in L^{2}
$$

if $\operatorname{Re} z>1-d / 2$,

$$
\left\|\mathcal{M}_{z} f\right\|_{\infty} \leqslant C_{z}\|f\|_{\infty} \quad \forall f \in L^{\infty}
$$

if $\operatorname{Re} z>0$, and

$$
\left\|\mathcal{M}_{z} f\right\|_{q, w} \leqslant C_{z}\|f\|_{q, w} \quad \forall f \in L^{q}(w)
$$

if $\operatorname{Re} z \geqslant 1$ and $w \in A_{q}$, when $q>1$. An iterated interpolation argument shows that, if $\theta_{1}, \theta_{2}, \theta_{3} \in[0,1]$ and $\theta_{1}+\theta_{2}+\theta_{3}=1$, then

$$
\left\|\mathcal{M}_{z} f\right\|_{p, w^{\delta}} \leqslant C_{z}\|f\|_{p, w^{\delta}} \quad \forall f \in L^{p}\left(w^{\delta}\right)
$$

for all $w$ in $A_{q}$, provided that

$$
\operatorname{Re} z>\theta_{1}\left(1-\frac{d}{2}\right)+\theta_{3}, \quad \frac{1}{p}=\frac{\theta_{1}}{2}+\frac{\theta_{3}}{q} \quad \text { and } \quad \delta=\frac{p \theta_{3}}{q} .
$$

The proof then consists of choosing the interpolation parameters appropriately. Indeed, to obtain a weighted inequality for a given $p$ in $(1, \infty)$, we must choose nonnegative $\theta_{1}$ and $\theta_{3}$ such that

$$
\theta_{1}+\theta_{3} \leqslant 1, \quad \theta_{1}\left(1-\frac{d}{2}\right)+\theta_{3}<0 \quad \text { and } \quad 0<\frac{1}{\theta_{3}}\left(\frac{1}{p}-\frac{\theta_{1}}{2}\right)<1 .
$$


The first of these three conditions allows us to choose $\theta_{2}$ to be $1-\theta_{1}-\theta_{3}$, while the second ensures that the final result holds for $\mathcal{M}_{0}$, and the third means that we can find $q$ in $(1, \infty)$ such that $1 / p=\theta_{1} / 2+\theta_{3} / q$. The three conditions together imply that $p$ must be bigger than $d /(d-1)$. It also turns out that for each $p$ in $(d /(d-1), \infty)$, there is a range of $\theta_{1}$ and $\theta_{3}$ which satisfy the conditions, and hence a range of results.

Now we come to the details. We divide our argument into several cases. First we consider the case where $\delta=(d-2) /(d-1)$ and $d /(d-1)<p<\infty$. Next we consider the case where $d /(d-1)<p<d$ and $\delta=1-p / d$ : this requires several subcases. Finally we consider the case where $d \leqslant p<\infty$ and $\delta=0$. An interpolation argument completes the proof of the theorem.

CASE 1. We show that, if $d /(d-1)<p<\infty$ and $\delta=(d-2) /(d-1)$, then

$$
\left\|\mathcal{M}_{0} f\right\|_{p, w^{\delta}} \leqslant C\|f\|_{p, w^{\delta}} \quad \forall f \in L^{p}\left(w^{\delta}\right) \quad \forall w \in A_{1}
$$

Take $w$ in $A_{1}$, and recall that there exists a positive $\varepsilon_{0}$ such that $w^{1+\varepsilon} \in A_{1}$ for all $\varepsilon$ in $\left[0, \varepsilon_{0}\right)$. Since $p>d /(d-1)$, we may choose a small positive $\varepsilon_{1}$ such that

$$
\frac{d}{p(d-1)}+2 \varepsilon_{1}<1 \quad \text { and } \quad \frac{\varepsilon_{1} p(d-1)}{d-2}<\frac{\varepsilon_{0}}{1+\varepsilon_{0}}
$$

Now take $\varepsilon_{2}$ in $\left(0, \varepsilon_{0}\right)$ such that

$$
\frac{\varepsilon_{2}}{1+\varepsilon_{2}}=\frac{\varepsilon_{1} p(d-1)}{d-2}
$$

Define $\theta_{1}, \theta_{2}, \theta_{3}$ by

$$
\theta_{3}=\frac{d-2}{p(d-1)}, \quad \theta_{1}=\frac{2}{p(d-1)}+2 \varepsilon_{1} \quad . \text { and } \quad \theta_{2}=1-\theta_{1}-\theta_{3}
$$

then $0<\theta_{1}, \theta_{2}, \theta_{3}<1$. Take $q$ to be $1+\varepsilon_{2}$; then $1<q<\infty$ and $w^{1+\varepsilon_{2}} \in A_{q}$. Now interpolate between the estimates

$$
\left\|\mathcal{M}_{z} f\right\|_{2} \leqslant C_{z}\|f\|_{2} \quad \forall f \in L^{2}
$$

if $\operatorname{Re} z>1-d / 2$,

$$
\left\|\mathcal{M}_{z} f\right\|_{\infty} \leqslant C_{z}\|f\|_{\infty} \quad \forall f \in L^{\infty}
$$

if $\operatorname{Re} z>0$, and

$$
\left\|\mathcal{M}_{z} f\right\|_{q, w^{1+\varepsilon_{2}}} \leqslant C_{z}\|f\|_{q, w^{1+\varepsilon_{2}}} \quad \forall f \in L^{q}(w)
$$

if $\operatorname{Re} z \geqslant 1$, to conclude that

$$
\left\|\mathcal{M}_{0} f\right\|_{p, w^{\delta}} \leqslant C_{z}\|f\|_{p, w^{\delta}} \quad \forall f \in L^{p}\left(w^{\delta}\right),
$$

where $\delta=(d-2) /(d-1)$. 
CASE 2. For this case, we introduce the notation $p^{*}$ for $p(d-2) /(d-p)$. We show that. if $d /(d-1)<p<d$, then the inequality

$$
\left\|\mathcal{M}_{0} f\right\|_{p, w} \leqslant C\|f\|_{p, w} \quad \forall f \in L^{p}(w)
$$

holds for all $w$ of the form $u_{1}^{1-p / d} u_{2}^{1-p(d-1) / d}$, where $u_{1}$ and $u_{2}$ lie in $A_{1}$. Equivalently, it suffices to show that

$$
\left\|\mathcal{M}_{0} f\right\|_{p, w} \leqslant C\|f\|_{p, w} \quad \forall f \in L^{p}(w) \quad \forall w \in A_{p^{*}}^{1-p / d} .
$$

We consider three subcases. First, if $p=2$, then $p^{*}=2$. If $w \in A_{2}$, then there exists some $\varepsilon_{0}$ in $(0,1)$ such that $w^{1+\varepsilon} \in A_{2}$ for all $\varepsilon$ in $\left[0, \varepsilon_{0}\right)$. By choosing $\theta$ slightly less than $1-2 / d$, we may arrange that $\theta\left(1+\varepsilon_{0}\right)>1-2 / d$ and $(1-\theta)(1-d / 2)+\theta<0$; by then choosing $\varepsilon$ appropriately in $\left(0, \varepsilon_{0}\right)$, we may arrange that $w^{1+\varepsilon} \in A_{2}$ and $\theta(1+\varepsilon)=1-2 / d$.

On the one hand, if $\operatorname{Re} z>1-d / 2$, then

$$
\left\|\mathcal{M}_{z} f\right\|_{2} \leqslant C_{z}\|f\|_{2} \quad \forall f \in L^{2}
$$

and on the other hand, if $\operatorname{Re} z \geqslant 1$, then

$$
\left\|\mathcal{M}_{z} f\right\|_{2, w^{1+e}} \leqslant C_{z}\|f\|_{2, w^{1+e}} \quad \forall f \in L^{2}\left(w^{1+\varepsilon}\right) .
$$

By interpolation,

$$
\left\|\mathcal{M}_{z} f\right\|_{2, w^{\theta(1+\varepsilon)}} \leqslant C_{z}\|f\|_{2, w^{\theta(1+\varepsilon)}} \quad \forall f \in L^{2}\left(w^{\theta(1+\varepsilon)}\right)
$$

provided that $\operatorname{Re} z>(1-\theta)(1-d / 2)+\theta$. The result claimed follows for this case.

Next, suppose that $d /(d-1)<p<2$, so that $1<p^{*}<p$. If $v \in A_{p^{*}}$, then there exists some $\varepsilon_{0}$ such that $w^{1+\varepsilon} \in A_{p^{*}}$ whenever $0<\varepsilon<\varepsilon_{0}$, and then there exists some $p^{* *}$ in $\left(1, p^{*}\right)$ such that $w^{1+\varepsilon} \in A_{p * *}$. Take $\theta$ and $\theta^{*}$ such that

$$
\frac{1}{p}=\frac{\theta}{p^{*}}+\frac{1-\theta}{2} \quad \text { and } \quad \frac{1}{p}=\frac{\theta^{*}}{p^{* *}}+\frac{1-\theta^{*}}{2} .
$$

As $p^{* *}$ approaches $p^{*}, \theta^{*}$ approaches $\theta$, so by taking $p^{* *}$ close enough to $p^{*}$ and $\varepsilon$ small enough, we may assume that

$$
\frac{(1+\varepsilon) p \theta^{*}}{p^{* *}}=\frac{p \theta}{p^{*}}
$$

Unravelling the definitions, we see that

$$
\theta=\frac{1 / p-1 / 2}{1 / p^{*}-1 / 2}=1-\frac{2}{d}
$$

and $\theta^{*}<\theta$, whence

$$
\theta^{*}+\left(1-\theta^{*}\right)\left(1-\frac{d}{2}\right)=1-\frac{d}{2}+\frac{d \theta^{*}}{2}<1-\frac{d}{2}+\frac{d \theta}{2}=0 .
$$


On the one hand, if $\operatorname{Re} z>1-d / 2$, then

$$
\left\|\mathcal{M}_{z} f\right\|_{2} \leqslant C_{z}\|f\|_{2} \quad \forall f \in L^{2},
$$

and on the other, if $\operatorname{Re} z \geqslant 1$, then

$$
\left\|\mathcal{M}_{z} f\right\|_{p^{* *}, w^{1+e}} \leqslant C_{z}\|f\|_{p^{* \cdot}, w^{1+e}} \quad \forall f \in L^{p^{* *}}\left(w^{1+\varepsilon}\right) .
$$

By interpolation,

$$
\left\|\mathcal{M}_{z} f\right\|_{p, w^{\delta}} \leqslant C_{z}\|f\|_{p, w^{\delta}} \quad \forall f \in L^{p}\left(w^{\delta}\right)
$$

if $\operatorname{Re} z>\theta^{*}+\left(1-\theta^{*}\right)(1-d / 2)$ and $\delta=(1+\varepsilon) p \theta^{*} / p^{* *}$; in particular, this inequality holds if $z=0$. It follows that

$$
\left\|\mathcal{M}_{0} f\right\|_{p, w^{\delta}} \leqslant C\|f\|_{p, w^{\delta}} \quad \forall f \in L^{p}\left(w^{\delta}\right)
$$

for all $w$ in $A_{p^{*}}$, where $\delta=1-p / d$, as claimed.

For the subcase where $2<p<d$, we argue in a similar fashion, taking $p^{* *}$ in $\left(p^{*}, \infty\right)$ and interpolating between the estimates (3.2) and (3.3) for this case.

Finally, if $d /(d-1)<p<d$, then another interpolation gives the intermediate cases enunciated.

CASE 3. We show that if $d \leqslant p<\infty$, then

$$
\left\|\mathcal{M}_{0} f\right\|_{p, w} \leqslant C\|f\|_{p, w} \quad \forall f \in L^{p}(w)
$$

for all $w$ of the form $u^{2-d}$, where $u \in A_{1}$.

Given $u$ in $A_{1}$, there exists some small positive $\varepsilon$ such that $u^{1+\varepsilon} \in A_{1}$. Choose $\delta_{1}$ and $\delta_{3}$ such that

$$
0<\theta_{1}=\frac{2}{p}-\delta_{1}<\frac{2}{p} \quad \text { and } \quad 0<\theta_{3}=\frac{d-2}{p}-\delta_{3}<\frac{d-2}{p}
$$

where $\delta_{3}>(d-2) \delta_{1} / 2$, and take $\theta_{2}$ to be $1-\theta_{1}-\theta_{3}$. If $\delta_{1}$ and $\delta_{3}$ are small enough, then $1<q<\infty$, and also

$$
\frac{(d-2) q}{p \theta_{3}(q-1)}=\left(1-\frac{p \delta_{3}}{d-2}\right)^{-1}\left(1-\frac{\delta_{1}}{2 \theta_{3}}\right)^{-1} \in(1,1+\varepsilon) .
$$

Now interpolate between the estimates

$$
\left\|\mathcal{M}_{z} f\right\|_{2} \leqslant C_{z}\|f\|_{2} \quad \forall f \in L^{2}
$$

if $\operatorname{Re} z>1-d / 2$,

$$
\left\|\mathcal{M}_{z} f\right\|_{\infty} \leqslant C_{z}\|f\|_{\infty} \quad \forall f \in L^{\infty}
$$

if $\operatorname{Re} z>0$, and

$$
\left\|\mathcal{M}_{z} f\right\|_{q, w} \leqslant C_{z}\|f\|_{q, w} \quad \forall f \in L^{q}(w)
$$


if $\operatorname{Re} z \geqslant 1$, where $w$ is chosen to be $u^{(2-d) q / p \theta_{3}}$, to conclude that

$$
\left\|\mathcal{M}_{0} f\right\|_{p, w} \leqslant C\|f\|_{p, w} \quad \forall f \in L^{p}(w)
$$

for all $w$ of the form $u^{2-d}$, where $u \in A_{1}$.

The results for intermediate values of $\delta$ for a given $p$ follow by interpolation from the extremal results with the same $p$.

Clearly our results improve the previous results of $[3,4,5]$, but do not reach the expected limit of $A_{1}^{1-1 / d}$.

\section{Estimates FOR FRACTIONAL POWERS OF THE LAPLACIAN}

For $\alpha$ in $[0, d]$, define $B_{\alpha}$ by the formula

$$
B_{\alpha}(\eta)=\pi^{-d / 2+\alpha+i \eta} \frac{\Gamma((d-\alpha-i \eta) / 2)}{\Gamma((\alpha+i \eta) / 2)},
$$

and write $K_{\alpha, \eta}$ for $B_{\alpha}(\eta)|\cdot|^{\alpha-d+i \eta}$. Note that $\widehat{K}_{\alpha, \eta}=|\cdot|^{-\alpha-i \eta}$ (see [15, p. 117]). The kernels $K_{\alpha, \eta}$ correspond to fractional (imaginary) powers of the Laplacian. In this section, we obtain weighted $L^{p}-L^{q}$ estimates for the kernels $K_{\alpha, \eta}$.

Before we consider weighted $L^{p}-L^{q}$ estimates, we remind the reader that, for $\xi$ and $\eta$ in $\mathbf{R}$,

$$
\frac{e^{\pi|\eta| / 2}|\Gamma(\xi+i \eta)|}{|\eta|^{\xi-1 / 2}} \longrightarrow(2 \pi)^{1 / 2}
$$

as $|\eta| \rightarrow \infty$ (see, for example, [18, p. 151]). Since $\Gamma$ is meromorphic, with simple poles at $0,-1,-2, \ldots$, it follows that, given $a, b$, and $c$ in $\mathbf{R}$, there exist constants $C_{1}$ and $C_{2}$ such that

$$
C_{1}|\eta|^{2 \xi+a-b} \leqslant\left|\frac{\Gamma(a+\xi+i \eta)}{\Gamma(b-\xi-i \eta)}\right| \leqslant C_{2}|\eta|^{2 \xi+a-b}
$$

for all $\xi$ in $[-c, c]$ and all $\eta$ in $\mathbf{R} \backslash(-1,1)$. The behaviour of the quotient when $\eta$ is small may be controlled using the poles of $\Gamma$.

THEOREM 4.1. Suppose that $1<t<\infty$, and that $\max \{0,2-t\}<\gamma<1$. Suppose also that $w$ is in $A_{s}$, where

$$
s=\frac{t+2 \gamma-2}{\gamma}
$$

Then there exist constants $C$ and $\varepsilon$ such that

$$
\left\|K_{0, \eta} * f\right\|_{t, w^{\gamma}} \leqslant C(1+|\eta|)^{E-\varepsilon}\|f\|_{t, w^{\gamma}} \quad \forall f \in L^{t}\left(w^{\gamma}\right)
$$

for all $\eta$ in $\mathbf{R}$, where $E=d s \gamma / 2 t$. 
Proof: Fix $t$ and $\gamma$, and hence $s$, as enunciated, so that $s>1$, and take $w$ in $A_{s}$. Let $\theta$ be $s \gamma / t$; then $0<\theta<1$.

On the one hand, by Fourier analysis,

$$
\left\|K_{0, \eta} * f\right\|_{2}=\|f\|_{2} \quad \forall f \in L^{2} .
$$

On the other hand, as shown in [4], for all small positive $\delta$ there exists a constant $C$ such that, for all $\eta$ in $\mathbf{R}$,

$$
\left\|K_{0, \eta} * f\right\|_{s, w} \leqslant C(1+|\eta|)^{d / 2+\delta}\|f\|_{s, w} \quad \forall f \in L^{s}(w) .
$$

Complex interpolation shows that

$$
\left\|K_{0, \eta} * f\right\|_{t, w^{\gamma}} \leqslant C(1+|\eta|)^{\theta(d / 2+\delta)}\|f\|_{t, w^{\gamma}} \quad \forall f \in L^{t}\left(w^{\gamma}\right),
$$

which is the required result, except that the exponent of $(1+|\eta|)$ is a little too big. Gunawan and Wright have recently shown that the term $\delta$ is not necessary in any of the above inequalities - details will appear elsewhere. However, this improved estimate is still not as strong as possible.

To improve (4.3), we recall that there exist $\zeta_{0}$ and $s_{0}$ in $\mathrm{R}^{+}$such that $1 \leqslant s_{0}<s$ and $w^{1+\zeta} \in A_{s}$ for all $\zeta$ in $\left[0, \zeta_{0}\right)$ and all $s$ in $\left(s_{0}, \infty\right)$. Choose $\theta^{*}$ and $s^{*}$ very close to $\theta$ and $s$ such that $\theta^{*}<\theta, s_{0}<s^{*}$,

$$
\theta^{*}\left(\frac{1}{s^{*}}-\frac{1}{2}\right)=\theta\left(\frac{1}{s}-\frac{1}{2}\right) \quad \text { and } \quad \frac{\left(1+\zeta_{0}\right) s \theta^{*}}{s^{*} \theta} \geqslant 1 .
$$

Now choose $\delta$ and $\zeta$ such that

$$
\theta^{*}(d / 2+\delta)<\theta d / 2 \quad \text { and } \quad \frac{(1+\zeta) s \theta^{*}}{s^{*} \theta}=1
$$

Interpolation between the estimates

$$
\left\|K_{0, \eta} * f\right\|_{s^{*}, w^{1+\zeta}} \leqslant C(1+|\eta|)^{d / 2+\delta}\|f\|_{s^{*}, w^{1+\varsigma}} \quad \forall f \in L^{s^{*}}\left(w^{1+\varsigma}\right)
$$

and

$$
\left\|K_{0, \eta} * f\right\|_{2}=\|f\|_{2} \quad \forall f \in L^{2}
$$

yields the inequality enunciated.

Now we interpolate between our "optimal" weighted $L^{t}-L^{t}$ estimate and the trivial $L^{1}-L^{\infty}$ estimate to obtain weighted $L^{p}-L^{q}$ estimates.

THEOREM 4.2. Suppose that $1<p<q<\infty$ and that $\max \left\{0,1-q / p^{\prime}\right\}<\gamma<1$. Suppose also that $w \in A_{s}$, where

$$
s=\frac{q+2 p^{\prime} \gamma-p^{\prime}}{p^{\prime} \gamma}
$$

Then there exist constants $C$ and $\varepsilon$ such that

$$
\left\|K_{\alpha, \eta} * f\right\|_{q, \omega^{\gamma}} \leqslant C(1+|\eta|)^{E_{0}-\varepsilon}\|f\|_{p, w^{p \gamma / q}} \quad \forall f \in L^{p}\left(w^{p \gamma / q}\right),
$$

where $E_{0}=d(p q-2 q+2 p \gamma) / 2 p q$. 
Proof; From Theorem 4.1, given $t$ in $(1, \infty)$ and $\gamma$ in $(0,1)$ such that $t+\gamma>2$, if

$$
s=\frac{t+2 \gamma-2}{\gamma}
$$

and $w \in A_{s}$, then there exist constants $C$ and $\varepsilon$ such that

$$
\left\|K_{0, \eta} * f\right\|_{t, w^{\gamma}} \leqslant C(1+|\eta|)^{E-\varepsilon}\|f\|_{t, w^{\gamma}} \quad \forall f \in L^{t}\left(w^{\gamma}\right),
$$

where $E=d s \gamma / 2 t$.

Furthermore, for all $x$ in $\mathbf{R}^{d}$ and $f$ in $L^{1}$,

$$
\left|K_{d, \eta} * f(x)\right| \leqslant \int_{\mathbf{R}^{d}}\left|K_{d, \eta}(x-y)\right||f(y)| \mathrm{d} y=\left|B_{d}(\eta)\right|\|f\|_{1} \quad \forall f \in L^{1},
$$

whence

$$
\left\|K_{d, \eta} * f\right\|_{\infty} \leqslant C(1+|\eta|)^{-d / 2}\|f\|_{1} \quad \forall f \in L^{1} .
$$

We interpolate between the estimates (4.4) and (4.5). To do this, we consider the family of operators $\left(T_{\zeta}\right)_{0 \leqslant \text { Re } \varsigma \leqslant 1}$, given by

$$
T_{\xi+i \eta} f=\frac{\Gamma(d-E+(E / 2+d / 4)(\xi+i \eta))}{\Gamma(d-(E / 2+d / 4)(\xi+i \eta))} K_{d \xi, d \eta} * f .
$$

This family is continuous in the strip of definition and analytic in its interior (say, in the topology of operators from the space of simple functions to the space of functions with the topology of convergence in measure). Further, for some small positive $\varepsilon$ and all $\eta$ in $\mathbf{R}$,

$$
\begin{aligned}
\left\|T_{i \eta} f\right\|_{t, w^{\gamma}} & \leqslant C\left|\frac{\Gamma(d-E+i(E / 2+d / 4) \eta)}{\Gamma(d-i(E / 2+d / 4) \eta)}\right|(1+|\eta|)^{E-\varepsilon}\|f\|_{t, w^{\gamma}} \\
& \leqslant C(1+|\eta|)^{-\varepsilon}\|f\|_{\ell, w^{\gamma}} \quad \forall f \in L^{t}\left(w^{\gamma}\right),
\end{aligned}
$$

while

$$
\begin{aligned}
\left\|T_{1+i \eta} f\right\|_{\infty} & \leqslant C\left|\frac{\Gamma(5 d / 4-E / 2+i(E / 2+d / 4) \eta)}{\Gamma(3 d / 4-E / 2-i(E / 2+d / 4) \eta)}\right|(1+|\eta|)^{-d / 2}\|f\|_{1} \\
& \leqslant C\|f\|_{1} \quad \forall f \in L^{1} .
\end{aligned}
$$

Stein's complex interpolation theorem (see, for example, [17, p. 205]) implies that, if

$$
0<\theta<1, \quad \frac{1}{p}=\frac{1-\theta}{t}+\frac{\theta}{1} \quad \text { and } \quad \frac{1}{q}=\frac{1-\theta}{t}+\frac{\theta}{\infty},
$$

then

$$
\left\|T_{\theta+i \eta} f\right\|_{q, w^{\gamma}} \leqslant C(1+|\eta|)^{-(1-\theta) \varepsilon}\|f\|_{p, w^{p \gamma / q}} \quad \forall f \in L^{p}\left(w^{p \gamma / q}\right),
$$


which in turn implies that

$$
\left\|K_{\alpha, \eta} * f\right\|_{q, w^{\gamma}} \leqslant C(1+|\eta|)^{E_{0}-\varepsilon^{\prime}}\|f\|_{p, w^{p \gamma / q}} \quad \forall f \in L^{p}\left(w^{p \gamma / q}\right)
$$

for some positive $\varepsilon^{\prime}$, where $E_{0}=d(p q-2 q+2 p \gamma) / 2 p q$.

It is now a question of sorting out which $t$ is compatible with a given $p$ and $q$ to obtain the result. We leave the details to the reader.

4.1. Weighted $L^{p}-L^{q}$ eStimates for $\mathcal{M}^{\alpha}$. We now present an approach to the maximal estimates for $\mathcal{M}^{\alpha}$, based on the calculations for the homogeneous kernels $|\cdot|^{-\alpha-i \eta}$ and the use of the Mellin transform. See, for example, [2] or [4] for similar applications of the method.

THEOREM 4.3. Suppose that $d /(d-1)<p<q<d$, that $\alpha=d / p-d / q$, and that $\max \left\{0,1-q / p^{\prime}\right\}<\gamma \leqslant 1-q / d$. Suppose also that $w$ is in $A_{s}$, where

$$
s=\frac{q+2 p^{\prime} \gamma-p^{\prime}}{p^{\prime} \gamma}
$$

Then there exists a constant $C$ such that

$$
\left\|\mathcal{M}^{\alpha} f\right\|_{q, w^{\gamma}} \leqslant C\|f\|_{p, w^{p \gamma / q}} \quad \forall f \in L^{p}\left(w^{p \gamma / q}\right) .
$$

Proof: We first outline the Mellin transform method, and then apply it to the case in hand.

Using the Mellin transform, we may write formally

$$
\mu_{1}(x)=\frac{1}{2 \pi \omega_{d-1}} \int_{\mathbf{R}}|x|^{\alpha-d+i \eta} \mathrm{d} \eta
$$

where $\omega_{d-1}$ is the area of the unit sphere. Indeed, for $u: \mathbf{R}^{d} \rightarrow \mathbf{C}$, define $\bar{u}: \mathbf{R}^{+} \rightarrow \mathbf{C}$ by the formula

$$
\bar{u}(t)=\int_{S^{d-1}} u\left(t x^{\prime}\right) \mathrm{d} \sigma\left(x^{\prime}\right)=\omega_{d-1} \int_{\mathbf{R}^{d}} u(t x) \mathrm{d} \mu_{1}(x),
$$

where $\sigma$ is surface measure on the sphere. Then,

$$
\begin{aligned}
\frac{1}{2 \pi} \int_{\mathbf{R}} \int_{\mathbf{R}^{d}} u(x)|x|^{\alpha-d+i \eta} \mathrm{d} x \mathrm{~d} \eta & =\frac{1}{2 \pi} \int_{\mathbf{R}} \int_{\mathbf{R}^{+}}\left[\bar{u}(t) t^{\alpha}\right] t^{i \eta-1} \mathrm{~d} t \mathrm{~d} \eta \\
& =\frac{1}{2 \pi} \int_{\mathbf{R}} \int_{\mathbf{R}}\left[\bar{u}\left(e^{s}\right) e^{s \alpha}\right] e^{i \eta s} \mathrm{~d} s \mathrm{~d} \eta .
\end{aligned}
$$

This is the inverse Fourier transform (in 0) of the Fourier transform of $s \mapsto \bar{u}\left(e^{s}\right) e^{s a}$, and is therefore equal to $\bar{u}\left(e^{0}\right) e^{0 \alpha}$, as required.

From the definition of $B_{\alpha}$ and $K_{\alpha, \eta}$ (see (4.1)), it follows that

$$
\mu_{1}=\int_{\mathbf{R}} A_{\alpha}(\eta) K_{\alpha, \eta} \mathrm{d} \eta
$$


where

$$
A_{\alpha}(\eta)=\frac{1}{2 \pi \omega_{d-1} B_{\alpha}(\eta)} .
$$

From (4.2), $A_{\alpha}(\eta)=\mathrm{O}\left((1+|\eta|)^{\alpha-d / 2}\right)$. Now we see that

$$
t^{\alpha} \mu_{t}=\int_{\mathbf{R}} A_{\alpha}(\eta) t^{-i \eta} K_{\alpha, \eta} \mathrm{d} \eta
$$

whence

$$
t^{\alpha} \mu_{t} * f=\int_{\mathbf{R}} A_{\alpha}(\eta) t^{-i \eta}\left(K_{\alpha, \eta} * f\right) \mathrm{d} \eta
$$

Consequently,

$$
\mathcal{M}^{\alpha} f \leqslant \int_{\mathbf{R}}\left|A_{\alpha}(\eta)\right|\left|K_{\alpha, \eta} * f\right| \mathrm{d} \eta
$$

and hence

$$
\left\|\mathcal{M}^{\alpha} f\right\|_{q, w} \leqslant \int_{\mathbf{R}}\left|A_{\alpha}(\eta)\right|\left\|K_{\alpha, \eta} * f\right\|_{q, w} \mathrm{~d} \eta .
$$

For the case in hand, we obtain weighted $L^{p}-L^{q}$ estimates for $\mathcal{M}^{\alpha} f$ provided that

$$
\int_{\mathbf{R}}(1+|\eta|)^{\alpha-d / 2}(1+|\eta|)^{E_{0}-\varepsilon^{\prime}} \mathrm{d} \eta<\infty,
$$

where $E_{0}$ is as defined in Theorem 4.2. This boils down to the condition that

$$
\alpha-\frac{d}{2}+E_{0} \leqslant-1
$$

that is,

$$
d\left(\frac{1}{2}-\frac{1}{p}+\frac{\gamma}{q}\right) \leqslant \frac{d}{2}-\frac{d}{p}+\frac{d}{q}-1,
$$

whence

$$
\gamma \leqslant 1-\frac{q}{d}
$$

Observe that this condition and the condition $\max \left\{0,1-q / p^{\prime}\right\}<\gamma<1$ of Theorem 4.2 are compatible if and only if

$$
1-\frac{q}{p^{\prime}}<1-\frac{q}{d} \quad \text { and } \quad 0<1-\frac{q}{d}
$$

these inequalities hold if and only if $p>d /(d-1)$ and $q<d$.

At this point, we have weighted $L^{p}-L^{q}$ inequalities for $\mathcal{M}^{\alpha}$ when $d /(d-1)<p$ $<q<d$ (corresponding to the upper triangle in Figure 1). We may interpolate once again to obtain nontrivial weighted $L^{p}-L^{q}$ estimates for $\mathcal{M}^{\alpha}$ when $p<q<(d-1) p$ and $q \geqslant d$ (corresponding to the lower triangle in Figure 1); this method also improves some of our estimates in the upper triangle. 


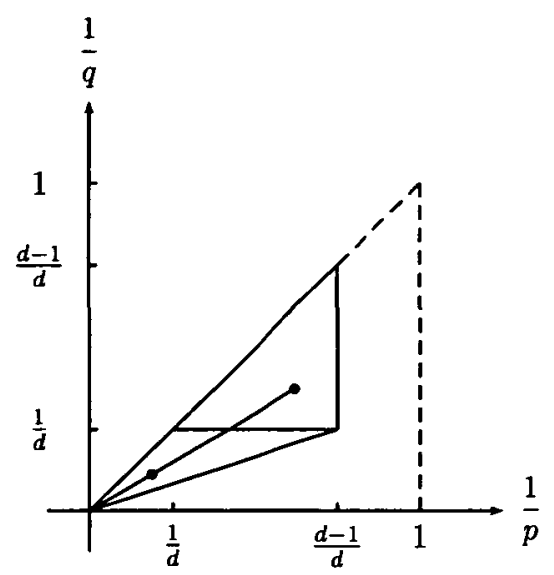

Figure 1:

TheOREM 4.4. Suppose that $d /(d-1)<p<q<(d-1) p$, that $\alpha=d / p-d / q$, and that $\max \{0,1-q / d\}<\gamma<1-q / p(d-1)$. Suppose also that $w$ is in $A_{s}$, where

$$
s=\frac{p[2 \gamma+d-d \gamma-1]-q}{p \gamma} .
$$

Then there exists a constant $C$ such that

$$
\left\|\mathcal{M}^{\alpha} f\right\|_{q, w^{\gamma}} \leqslant C\|f\|_{p, w^{p \gamma / q}} \quad \forall f \in L^{p}\left(w^{p \gamma / q}\right) .
$$

Proof: Given $p$ and $q$ such that $d /(d-1)<p<q<(d-1) p$, there exists $\theta$ in $(0,1)$ such that

$$
d /(d-1)<p \theta<q \theta<d
$$

If moreover

$$
\max \left\{0,1-q \theta /(p \theta)^{\prime}\right\}<\gamma \leqslant 1-q \theta / d
$$

and $w \in A_{s_{\theta}}$, where

$$
\theta_{\theta}=\frac{q \theta+2(p \theta)^{\prime} \gamma-(p \theta)^{\prime}}{(p \theta)^{\prime} \gamma}=\frac{2 p \gamma+p q \theta-q-p}{p \gamma}
$$

then we may interpolate between the inequalities

$$
\left\|\mathcal{M}^{0} f\right\|_{\infty} \leqslant C\|f\|_{\infty} \quad \forall f \in L^{\infty}
$$

(which is trivial) and

$$
\left\|\mathcal{M}^{\alpha / \theta} f\right\|_{q \theta, w^{\gamma}} \leqslant C\|f\|_{p \theta, w^{(p \theta) \gamma /(q \theta)}} \quad \forall f \in L^{p \theta}\left(w^{(p \theta) \gamma /(q \theta)}\right),
$$


(from Theorem 4.3) to obtain the inequality

$$
\left\|\mathcal{M}^{\alpha} f\right\|_{q, w^{\gamma}} \leqslant C\|f\|_{p, w^{p} / q} \quad \forall f \in L^{p}\left(w^{p \gamma / q}\right) .
$$

For given $p, q$ and $\gamma$, there are two questions to be answered: Does there exist $\theta$ in $(0,1)$ such that inequalities $(4.6)$ and $(4.7)$ both hold? And if so, what is the optimal $A_{s_{\theta}}$ for the weight $w$ in (4.8)?

We need to be able to choose $\theta$ such that

$$
p \theta>\frac{d}{d-1}, \quad q \theta<d, \quad 1-\frac{q \theta}{(p \theta)^{\prime}}<\gamma \quad \text { and } \quad \gamma \leqslant 1-q \theta / d .
$$

These inequalities boil down to the inequalities

$$
\theta>\frac{d}{p(d-1)}, \quad \theta<\frac{d}{q}, \quad \theta>\frac{1-\gamma}{q}+\frac{1}{p} \quad \text { and } \quad \theta \leqslant \frac{d(1-\gamma)}{q} .
$$

Thus such a $\theta$ exists if and only if

$$
\max \left\{\frac{d}{p(d-1)}, \frac{1-\gamma}{q}+\frac{1}{p}\right\}<\frac{d(1-\gamma)}{q}
$$

that is, if and only if $\gamma<1-q / p(d-1)$, which holds by hypothesis.

The best result is obtained by maximising $s_{\theta}$ subject to the conditions above, that is, taking $\theta$ equal to $d(1-\gamma) / q$. The condition that $\gamma>1-q / d$ in the statement of the theorem ensures that $d(1-\gamma) / q<1$.

\section{REFERENCES}

[1] J. Bourgain, 'Averages in the plane over convex curves and maximal operators', J. Analyse Math. 47 (1986), 69-85.

[2] M. Cowling and G. Mauceri, 'On maximal functions', Rend. Sem. Mat. Fis. Milano 49 (1979), 79-87.

[3] J. Duoandikoetxea and L. Vega, 'Spherical means and weighted inequalities', $J$. London Math. Soc. 53 (1996), 343-353.

[4] H. Gunawan, 'On weighted estimates for Stein's maximal function', Bull. Austral. Math. Soc. 54 (1996), 35-39.

[5] H. Gunawan, 'Some weighted estimates for Stein's maximal function', Bull. Malaysian Math. Soc. 21 (1998), 101-105.

[6] J. Garcia-Cuerva and J.-L. Rubio de Francia, Weighted norm inequalities and related topics (North-Holland, Amsterdam, 1985).

[7] G.H. Hardy and J.E. Littlewood, 'A maximal theorem with function-theoretic applications', Acta Math. 54 (1930), 81-116.

[8] E. Harboure, R.A. Macias and C. Segovia, 'Extrapolation results for classes of weights', Amer. J. Math. 110 (1988), 383-397. 
[9] B. Muckenhoupt, 'Weighted norm inequalities for the Hardy maximal function', Trans. Amer. Math. Soc. 165 (1972), 207-227.

[10] B. Muckenhoupt and R. Wheeden, 'Weighted norm inequalities for fractional integrals', Trans. Amer. Math. Soc. 192 (1974), 261-275.

[11] D.M. Oberlin, 'Operators interpolating between Riesz potentials and maximal operators', Ilinois J. Math. 33 (1989), 143-152.

[12] J.-L. Rubio de Francia, 'Weighted norm inequalities for homogeneous families of operators', Trans. Amer. Math. Soc. 275 (1983), 781-790.

[13] E.T. Sawyer, 'Weighted norm inequalities for fractional maximal operators', CMS Conf. Proc. 1 (1981), 283-309.

[14] E.T. Sawyer, 'A characterization of a two weight norm inequality for maximal operators', Studia Math. 75 (1982), 1-11.

[15] E.M. Stein, Singular integrals and differentiability properties of functions (Princeton Univ. Press, Princeton, N.J., 1970).

[16] E.M. Stein, 'Maximal functions: spherical means', Proc. Nat. Acad. Sci. U.S.A. 73 (1976), 2174-2175.

[17] E.M. Stein and G. Weiss, Introduction to Fourier analysis on Euclidean spaces (Princeton Univ. Press, Princeton, N.J., 1971).

[18] E.C. Titchmarsh, The theory of functions, (2nd edition) (Oxford Univ. Press, New York, 1939).

Michael Cowling

School of Mathematics

University of New South Wales

Sydney NSW 2052

Australia

H. Gunawan

Jurusan Matematika

Institut Teknologi Bandung

Bandung 40132

Indonesia
José García-Cuerva

Departamento de Matemáticas, C-XV

Universidad Autónoma de Madrid

28049-Madrid

Spain 